\title{
Blueberry fruit drop-associated virus: A New Member of the Family Caulimoviridae Isolated From Blueberry Exhibiting Fruit-Drop Symptoms
}

Alfredo Diaz-Lara, Department of Botany and Plant Pathology, Oregon State University, Corvallis, OR 97331; and Robert R. Martin, USDA-ARS, Horticultural Crops Research Unit, Corvallis, OR 97330

\begin{abstract}
Diaz-Lara, A., and Martin, R. R. 2016. Blueberry fruit drop-associated virus: A new member of the family Caulimoviridae isolated from blueberry exhibiting fruit drop symptoms. Plant Dis. 100:2211-2214.

This study describes the nucleotide sequence and genome organization of a new DNA virus isolated from 'Bluecrop' blueberry plants exhibiting fruit-drop symptoms and named Blueberry fruit drop-associated virus $(\mathrm{BFDaV})$. Blueberry fruit drop disease was first detected in blueberry plants in British Columbia, Canada in the late 1990s, and in a single field in northern Washington state in the United States in 2012. Infected bushes abort nearly $100 \%$ of their fruit about three weeks prior to harvest, when the berries are about 3 to $5 \mathrm{~mm}$ in diameter. At harvest, the affected plants appear taller than healthy ones as there is no fruit weighing down

the branches. The virus was amplified from diseased material using rolling circle amplification, followed by enzyme digestion, cloning, and sequencing. The full genome of $\mathrm{BFDaV}$ is $9,850 \mathrm{bp}$ in length and contains a single open reading frame, encoding for a polyprotein, and a large noncoding region. Based on the genome size and organization and phylogenetics, BFDaV is proposed as a new and the largest member of family Caulimoviridae. Finally, in mapping part of a field with fruit-drop symptoms, there was a nearly perfect correlation between the presence of the virus and fruit-drop symptoms.
\end{abstract}

The Pacific Northwest (Oregon and Washington in the United States and British Columbia in Canada) is the world's largest blueberry (Vaccinium corymbosum; family Ericaceae) production area with greater than 175,000 million pounds produced in 2014 (http:// www.bcblueberry.com/; https://www.nass.usda.gov/Data_and_ Statistics/). Recently, a fruit-drop symptom has been observed in several fields of 'Bluecrop', in the Fraser River Valley in northwest Washington state and southwest British Columbia, Canada. Also, it was observed that young leaves exhibited a transient red coloration of the veins during the bloom period and the corolla of the flowers exhibited some red striping. After bloom, the plants appear normal until about three weeks prior to harvest, when the fruit drops (Fig. 1). Prior to harvest, affected bushes can be identified easily, since they stand upright, as there is no fruit weighing down the branches. Several studies failed to identify any fungi, bacteria, or virus related to the new disorder (Martin et al. 2006). Later, a cryptic virus (Blueberry latent virus) was characterized but was not associated with this or any disease in blueberry and it was widespread in all production blueberry areas, in multiple cultivars of highbush blueberry, and in multiple species of Vaccinium (Martin et al. 2011).

In this paper, we describe the genome of Blueberry fruit dropassociated virus (BFDaV), a new member of the family Caulimoviridae, whose properties differ from other genera in the family and provide evidence that it is the causal agent of the disease.

\section{Materials and Methods}

Plant material. Leaves were collected from blueberry 'Bluecrop' plants that exhibited fruit-drop symptoms and from asymptomatic

Corresponding author: Alfredo Diaz-Lara, Email: diazlara@oregonstate.edu

Nucleotide sequence data has been deposited in the GenBank database under accession number KT148886.

*The $\boldsymbol{e}$-Xtra logo stands for "electronic extra" and indicates that two supplementary tables are published online.

Accepted for publication 1 July 2016.

http://dx.doi.org/10.1094/PDIS-06-16-0792-RE

This article is in the public domain and not copyrightable. It may be freely reprinted with customary crediting of the source. The American Phytopathological Society, 2016. plants during 2014 and 2015 growing seasons from commercial fields in the state of Washington in the United States (one field) and British Columbia, Canada (two fields). The plant material, used for nucleic acid extractions and cloning, was tested for all known viruses of blueberry and found to be infected only with Blueberry latent virus (BBLV).

DNA extraction, rolling circle amplification (RCA), enzymatic digestion, cloning, and resequencing. Total DNA was extracted from leaf tissue as described by Lockhart (1990), with the addition of a proteinase $\mathrm{K}$ digestion prior to the nucleic acid extractions with phenol-chloroform-isoamyl alcohol (25:24:1) and chloroformisoamyl alcohol (24:1). Purified DNA was used as template for RCA, using the Illustra TempliPhi 500 amplification kit (GE Healthcare, Buckinghamshire, U.K.) and following the manufacturer's instructions. RCA exponentially amplifies single- or double-stranded circular DNA via random hexamer primers and Phi29 DNA polymerase (Dean et al. 2001; Lizardi et al. 1998). The RCA product then was digested with EcoRI (New England Biolabs, Ipswich, MA, U.S.A.) and was separated using agarose gel electrophoresis. The EcoRIdigestion resulted in several restriction fragments, which were cloned into pBluescript II KS(+) vector using Quick T4 DNA ligase (New England Biolabs, Ipswich, MA, U.S.A.) and were transformed into One Shot Top 10 chemically competent cells of Escherichia coli (Invitrogen Corp., Carlsbad, CA, U.S.A.). Plasmids were sequenced, using M13 primers, followed by primer walking, and finally, primers were designed and used to sequence across the EcoRI restriction sites (Supplementary Table S1). Sequences were assembled using Geneious v4.8 (Kearse et al. 2012) to produce a preliminary sequence of BFDaV. In order to confirm the $\mathrm{BFDaV}$ sequence, additional primers were designed that overlapped and the entire genome of BFDaV was resequenced threefold developing a consensus sequence.

Genomic analysis of BFDaV. Nucleotide (nt) and translated amino acid (aa) sequence of BFDaV were compared with existing sequences in GenBank. ORF Finder (https://www.ncbi.nlm.nih.gov/ orffinder) was used to identify open reading frames (ORFs), which were also analyzed independently using BLASTp (Gish and States 1993) and the Conserved Domain search tool (Marchler-Bauer and Bryant 2004). Also, aa sequences were aligned and the pairwise scores were calculated using ClustalW2 (Larkin et al. 2007) and PIR (protein information resource) (http://pir.georgetown.edu/ pirwww/search/comp_mw.shtml) was employed to calculate the molecular mass of the polyprotein. Nuclear export signals and cleavage sites of protease were predicted using NetNES1.1 (la Cour et al. 
2004) and PROSPER (Song et al. 2012), respectively. Patterns and profiles were predicted using ELM (Dinkel et al. 2013). Finally, PLACE (Higo et al. 1999) was used to search for plant cis-acting regulatory DNA elements.

Phylogenetic analysis. The phylogenetic analysis of BFDaV was performed in Mesquite (http://mesquiteproject.org) and RAxML (Stamatakis 2014), using the JTT aa model of substitution and was confirmed with a bootstrap of 100 pseudoreplicates. A maximum likelihood tree was obtained, based on the aa sequence alignment of reverse transcriptase (RT) present in different members of the family Caulimoviridae (viruses and the accession numbers are listed in Figure 2). The phylogram was visualized in FigTree v1.4 (http:// tree.bio.ed.ac.uk/software/figtree) and shows all clades with a bootstrap value $50 \%$ or greater.

Virus detection. Specific primers (FruitdropFr1F1: 5'-GACAACAG CATCTACATCTCTGC-3' and FruitdropFr1R1: 5'-GGTCGTTCTAC CACGTTTGTG- $3^{\prime}$ ) that flank conserved regions of the virus aspartate protease and amplify a $395 \mathrm{bp}$ amplicon were designed to test for BFDaV in plant tissue by polymerase chain reaction (PCR). DNA extracts previously obtained were used as templates for the PCR amplification, using Taq polymerase (GenScript, Piscataway, NJ, U.S.A.) and a program consisting of $3 \mathrm{~min}$ at $95^{\circ} \mathrm{C}, 40$ cycles of $40 \mathrm{~s}$ at $95^{\circ} \mathrm{C}, 40 \mathrm{~s}$ at $53^{\circ} \mathrm{C}, 40 \mathrm{~s}$ at $72^{\circ} \mathrm{C}$, and a final elongation step of $7 \mathrm{~min}$ at $72^{\circ} \mathrm{C}$.

\section{Results}

Plants in the three fields studied tested negative for Blueberry leaf mottle, Blueberry scorch, Blueberry shoestring, Blueberry shock, Peach rosette mosaic, Tobacco ringspot, and Tomato ringspot viruses by enzyme-linked immunosorbent assay (ELISA) and for Blueberry red ringspot virus and Blueberry virus $A$ by RT-PCR. The plants did test positive for BBLV, which has not been associated with any symptoms in Vaccinium species. These results suggested the possibility that an unknown agent was responsible for the observed fruit-drop symptoms.

Virus characterization. Three restriction fragments (bands) were observed in the agarose gel and were later fully sequenced (Fig. 3A). The two larger restriction fragments of the RCA products encompassed the entire viral genome; furthermore, only two EcoRI recognition sites (GAATTC) were identified in the BFDaV genome, confirming accuracy of the restriction profile from the RCA product. The third band from the EcoRI-digested RCA reaction (1,281 bp) aligned with chloroplast sequence from blueberry and was not present in the resequenced BFDaV.
The complete genomic sequence of BFDaV consisted of 9,850 bp (GenBank accession number KT148886), making it the largest known member in the family Caulimoviridae. The intergenic region (IR) of BFDaV was 2,056 bp and contained different plant cis-acting regulatory DNA elements, such as five GATA boxes and one TATA box; these promoter elements were identified previously in other Caulimoviridae viruses (Lam and Chua 1989; Medberry et al. 1992). A plant tRNA ${ }^{\text {Met }}$ (TGGTATCAGAGC $1-12$ ) and priming site for RT was predicted within the IR (Medberry et al. 1990). This noncoding RNA was chosen as the starting location for the circular genome, as has been done for other caulimoviruses (Bouhida et al. 1993; Laney et al. 2012).

The nt analysis identified similarity between BFDaV and a reduced number of members of family Caulimoviridae. The genome of BFDaV shared $66 \%$ nt sequence identity with Strawberry vein banding virus (SVBV) and Cauliflower mosaic virus (CaMV) but

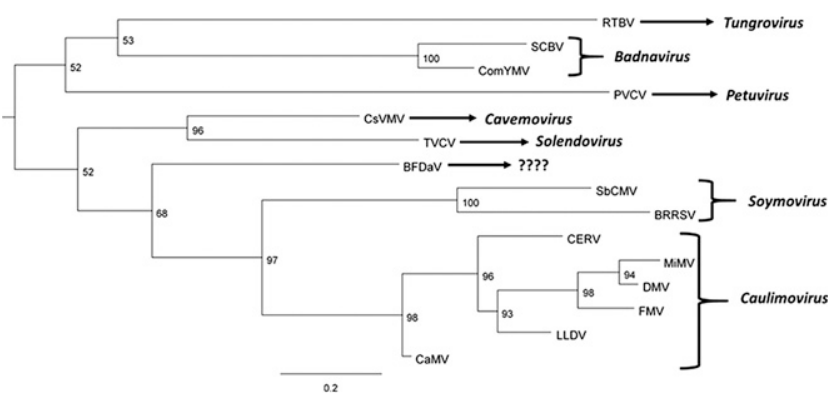

Fig. 2. Phylogenetic inference of Blueberry fruit drop-associated virus (BFDaV) in relation to members of the family Caulimoviridae: Cauliflower mosaic virus (CaMV; M90543.1), Carnation etched ring virus (CERV; NC_003498.1), Lamium leaf distortion-associated virus (LLDV; NC_010737.1), Figwort mosaic virus (FMV; NC_003554.1), Dahlia mosaic virus (DMV; NC_018616.1), Mirabilis mosaic virus (MiMV; NC_004036.1), Blueberry red ringspot virus (BRRSV; NC_003138.2), Soybean chlorotic mottle virus (SbCMV; NC_001739.2), BFDaV (KT148886), Cassava vein mosaic virus (CsVMV; NC_001648.1), Tobacco vein clearing virus (TVCV; NC_003378.1), Petunia vein clearing virus (PVCV; NC_001839.2), Rice tungro bacilliform virus (RTBV; D10774.1), Sugarcane bacilliform virus (SCBV; NC_013455.1), and Commelina yellow mottle virus (ComYMV; NC_001343.1) Maximum likelihood tree based on the amino acid sequences of the conserved reverse transcriptase, using the JTT model of substitution. Horizontal branch length is proportional to genetic distance; the scale bars represent changes per site. Bootstrap values less than $50 \%$ are not shown.
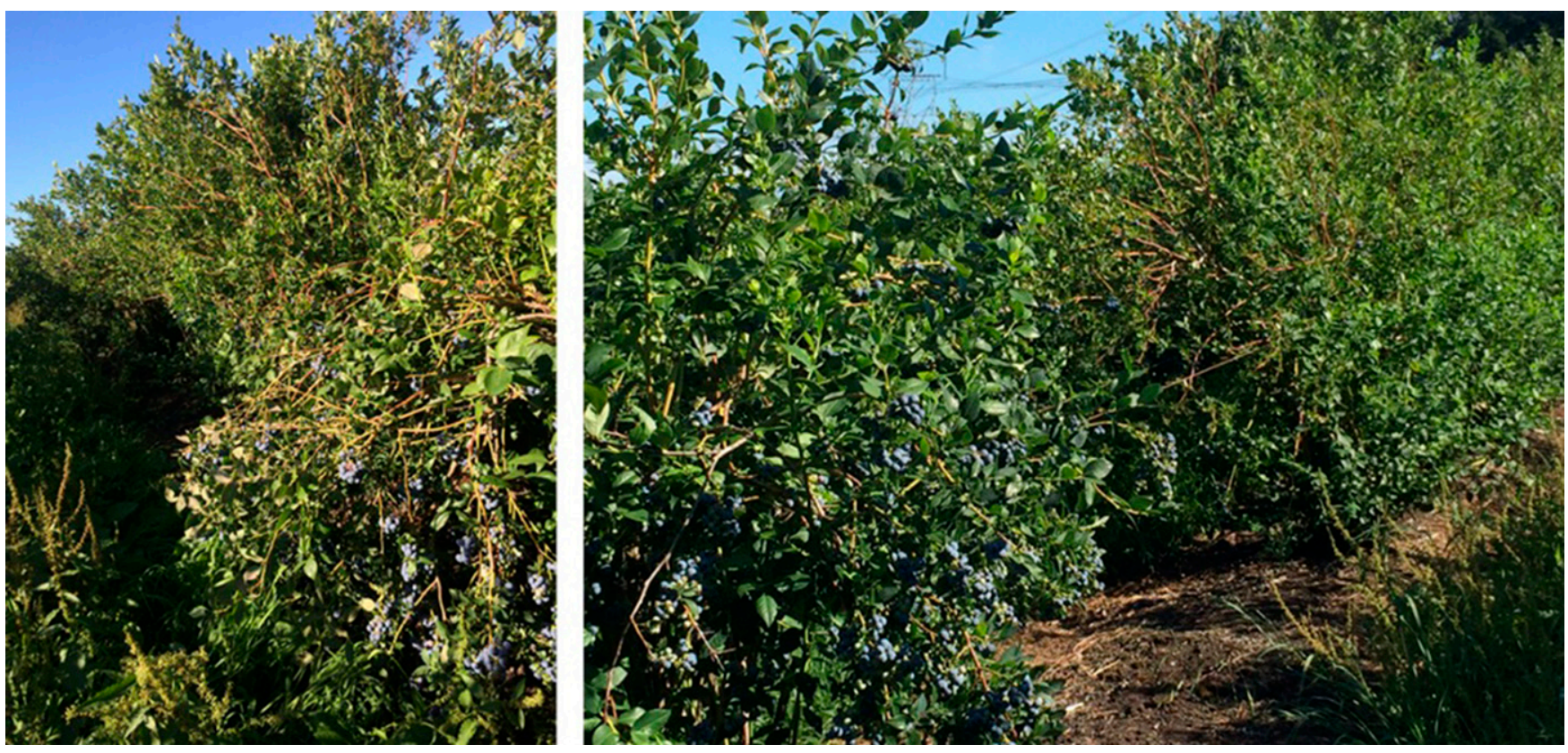

Fig. 1. Symptoms of fruit abortion. Healthy plant with normal fruit load in the foreground, fruit-drop plants in the background without any fruit. 
only over a small portion of the genome (4 and 5\% respectively), the rest of the BFDaV genome does not match with any identified viral sequence.

Unlike SVBV and CaMV, the new species contains a single ORF encoding for a large polyprotein (Fig. 4). Homology was observed between aa sequences of the BFDaV polyprotein and SVBV ORF 5 (50\% aa identities), Rudbeckia flower distortion virus (RuFDV) putative enzymatic polyprotein ( $48 \%$ aa identities), and Horseradish latent virus polyprotein ( $45 \%$ aa identities), only over $24 \%$ of the

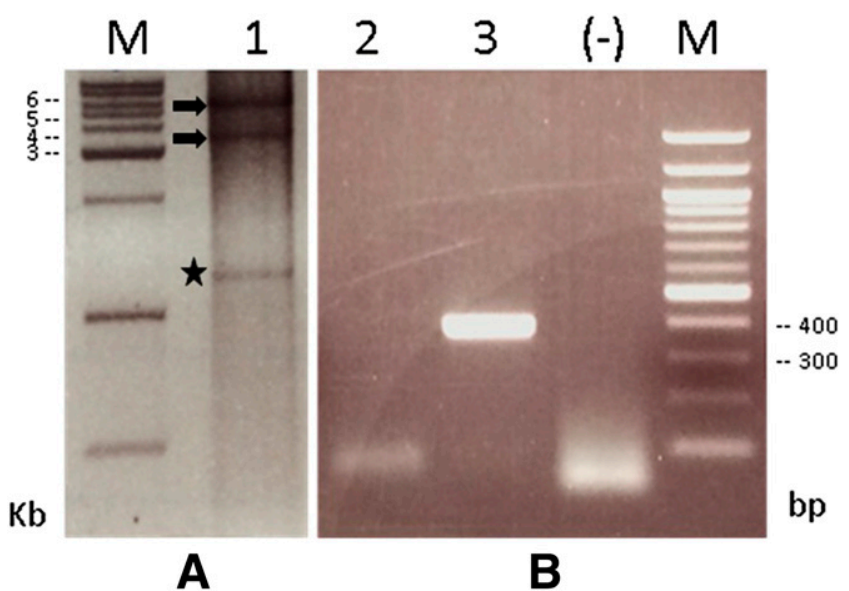

Fig. 3. A, Restriction profile of Blueberry fruit drop-associated virus (BFDaV). Lane 1, The entire genome of BFDaV, amplified with rolling circle amplification and digested with EcoRl enzyme, arrows indicate restriction fragments (6.2 and $3.6 \mathrm{~kb}$ ) belonging to BFDaV; the star indicates blueberry chloroplast sequence; lane M:, 1-kb ladder. B, Amplicons of BFDaV from blueberry 'Bluecrop', using detection primers. Lane 2, Asymptomatic plant; lane 3, symptomatic plant; lane (-), water control, expected amplicon size 395 bp; lane M, 100-bp ladder. polyproteins. Except for RuFDV (unclassified genus), the viruses mentioned that exhibit some homology to BFDaV belong to the genus Caulimovirus, whose genomes contain six or seven ORFs and contrast with the single polyprotein identified in BFDaV. This polyprotein of BFDaV has a length of 2,597 aa and a molecular mass of $301.6 \mathrm{kDa}$ and contains the following signature domains: a movement protein ( Ile $_{327}-\mathrm{Ser}_{446}$ ), previously identified and characterized from CaMV (Kasteel et al. 1996); a zinc knuckle (Cys997-Pro ${ }_{1011}$ ), which is a zinc binding motif $\mathrm{CX}_{2} \mathrm{CX}_{4} \mathrm{HX}_{4} \mathrm{C}$ (where $\mathrm{X}$ can be any aa) from retroviral gag proteins (nucleocapsid) (Zhou et al. 2007); pepsin-like aspartate protease $\left(\mathrm{Leu}_{1380^{-}}-\mathrm{Phe}_{1461}\right)$; RT $\left(\mathrm{Lys}_{1752^{-}}\right.$ Gly $_{1902}$ ) responsible for the replication of retroelements, including caulimoviruses (Xiong and Eickbush 1990) and a ribonuclease $\mathrm{H}$ (RNase H) ( $\left.\mathrm{Val}_{2000}-\mathrm{Arg}_{2122}\right)$. Additionally, a coiled-coil region was found upstream of the zinc finger domain as well as one nuclear export signal. Based on previous information and identification of several protease cleavage sites, it is suggested that the BFDaV polyprotein is posttranslationally cleaved to yield a movement protein, a coat protein, and a replicase, which are key components of most plant pararetroviruses.

Taxonomic placement. Phylogenetic analyses placed BFDaV within the family Caulimoviridae but distinct from the currently recognized genera (Fig. 2). In addition, the new virus is evolutionarily distant from Petunia vein clearing virus (PVCV), another species in the family Caulimoviridae, genus Petuvirus, with a single ORF (Richert-Pöggeler and Shepherd 1997). Although the genomic arrangement of BFDaV is similar to the Petuvirus sp., no sequence similarities were identified between BFDaV and the Petuvirus using BLASTn and BLASTx, and a ClustalW2 alignment between polyproteins of PVCV and BFDaV produced a low pairwise score (14.82\%). Additionally, the genome of BFDaV is 2,644 bp larger than PVCV.

Virus detection and diversity. During the summer of 2014 and spring of 2015, a total of 344 plants were tested via PCR, resulting

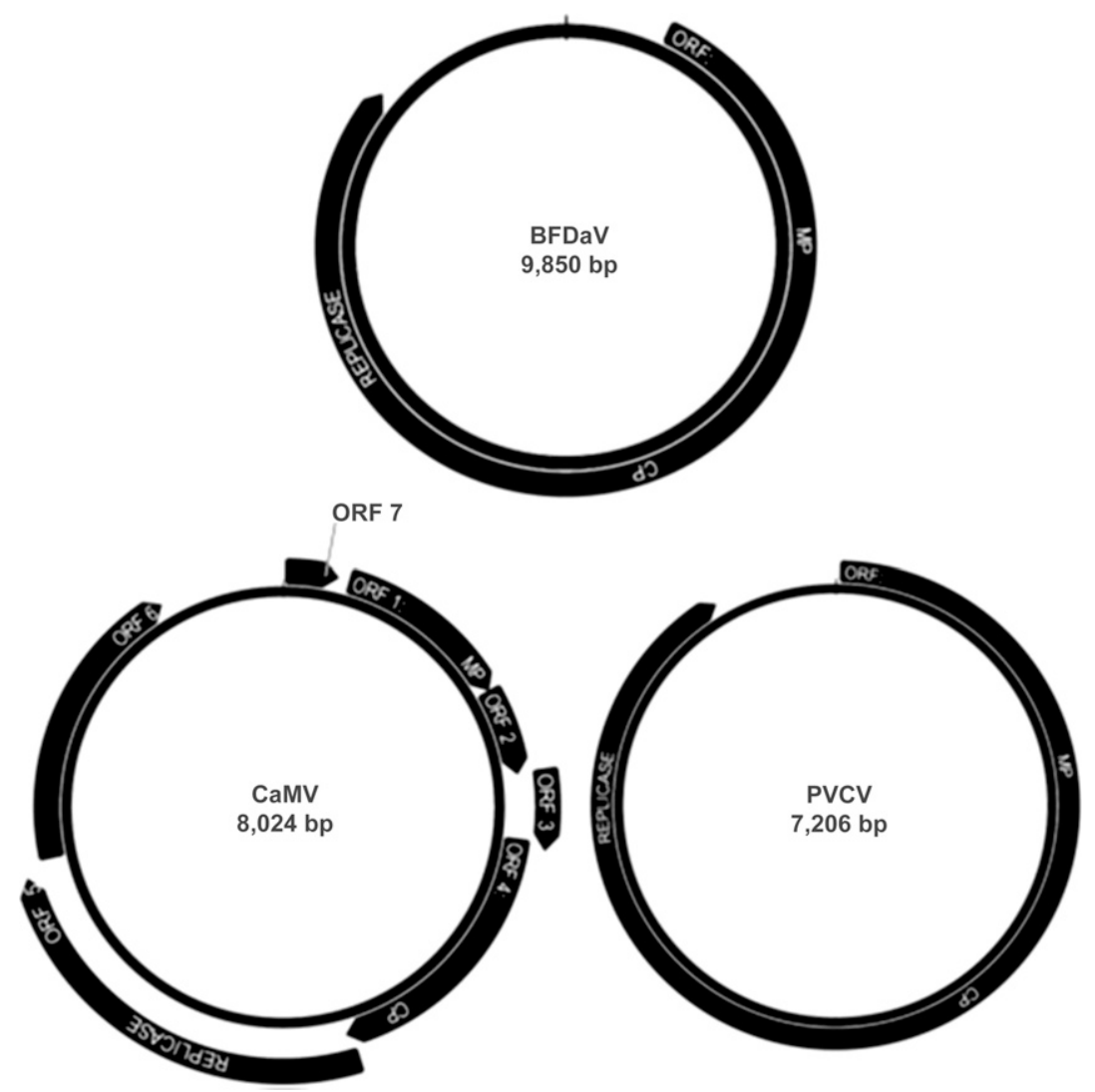

Fig. 4. Genomic organization of Blueberry fruit drop-associated virus (BFDaV), Cauliflower mosaic virus (CaMV, genus Caulimovirus), and Petunia vein clearing virus (PVCV, genus Petuvirus). ORF, open reading frame; MP, movement protein; and CP, coat protein. 
in 71 positive results for BFDaV (data not shown). The infected samples were collected in three different blueberry fields in the Fraser River Valley that were separated by 50 to $60 \mathrm{~km}$ from each other. Subsequently, the RNase $\mathrm{H}$ and coat protein of an isolate of BFDaV from each of the three fields were sequenced and compared (data not shown) to estimate the virus diversity, the three sources were very similar, having nt sequence identities ranging from 96 to $100 \%$ in the case of the RNase $\mathrm{H}$ and 92 to $93 \%$ for the coat protein. This is a limited number of isolates, but at this time these are the only three fields identified with fruit-drop symptoms.

To examine the relationship of BFDaV with symptoms observed in the field, a section of the field with approximately $50 \%$ incidence of fruit-drop symptoms was used. Sixty-six plants in a single row of blueberries in the field in Washington state were evaluated for virus symptoms and tested for BFDaV by PCR in the spring and summer of 2015 (Supplementary Table S2). In spring (mid-May) there were 34 plants that exhibited vein reddening in the young leaves, 31 plants without any vein reddening, and one plant that was not rated, since it had symptoms of Blueberry shock virus (BIShV), in which most leaves were necrotic and subsequently tested positive for BIShV by ELISA. The 34 symptomatic plants as well as the plant with BlShV were found positive for BFDaV by PCR and the 31 asymptomatic plants were negative. The 34 plants with leaf vein reddening symptoms in the spring exhibited fruit-drop symptoms prior to harvest in July and tested positive for BFDaV, when tested using the detection primers in PCR assays in July. The 31 asymptomatic plants in the spring did not show any fruit-drop symptoms in the summer and they all tested negative for BFDaV in PCR assays in July. The one plant that had symptoms of BlShV was not rated for fruit-drop symptoms, since BlShV causes a severe flower necrosis and there was very little fruit produced on the plant, but it was positive for $\mathrm{BFDaV}$ in July.

Fields of 'Duke', 'Liberty', and 'Bluejay' growing adjacent to infected blocks of 'Bluecrop' did not exhibit any fruit-drop symptoms and tested negative for BFDaV in PCR assays of 96 plants.

\section{Discussion}

There are eight genera (Caulimovirus, Badnavirus, Cavemovirus, Petuvirus, Solendovirus, Soymovirus, Tungrovirus, and Rosadnavirus) in the family Caulimoviridae, but BFDaV did not have a level of sequence similarity that placed it in any of the eight genera (Geering and Hull 2012). Even considering the low sequence homology to known members of the genus Caulimovirus, the genome arrangement of the new virus was similar to that of genus Petuvirus (Fig. 4). Consequently, we propose that BFDaV represents a novel genus in this family.

Using RCA and, later, detection primers (PCR), we confirmed the presence of the virus from symptomatic plants but not from healthy plants (Fig. 3), supporting the hypothesis that BFDaV is the cause of the fruit-drop symptom observed in 'Bluecrop' blueberry. Also, there was an excellent correlation between the presence of BFDaV and the fruit-drop symptoms, which supports the assertion that $\mathrm{BFDaV}$ is the causal agent of the syndrome. At the present time, this disease is restricted to a few commercial fields in the Fraser River Valley in southwestern British Columbia and northwest Washington state. Measures are to be taken to limit the distribution of this virus and prevent it entering nurseries and germplasm repositories. PCR testing for $\mathrm{BFDaV}$ has been added to G1 (top tier) plants in the National Clean Plant Network. Additionally, efforts are underway to determine if this virus can be detected in native vegetation in the Fraser River Valley adjacent to fields where the virus has been detected and to work with growers to eradicate this virus from production fields. BFDaV has been detected only in blueberry 'Bluecrop' and not in several other blueberry fields, ranging from 5 to 35 years old, adjacent to infected blocks with infected 'Bluecrop' plants. Graft transmission studies to other blueberry cultivars is underway.

Finally, mechanical transmissions were attempted from flower tissue and young leaves of symptomatic blueberry plants to several species of herbaceous host indicator plants (Cucurbita pepo, Glycine max, Nicotiana occidentalis, $N$. tabacum, $N$. sylvestris, $N$. benthamiana,
Cucumis sativus, Phaseolus vulgaris, Chenopodium amaranticolor, Chenopodium quinoa, Beta vulgaris, Solanum lycopersicum, and Brassica rapa). All attempts at mechanical transmission from blueberry plants exhibiting the fruit-drop symptom have been unsuccessful.

\section{Acknowledgments}

This work was funded by the United States Department of Agriculture Agricultural Research Service, Oregon Blueberry Commission, Washington Blueberry Commission, BC Blueberry Industry Development Council, National Clean Plant Network, and APHIS Plant Pest and Disease Management and Disaster Prevention Program. We thank D. F. Quito-Avila from the Centro de Investigaciones Biotecnológicas del Ecuador-ESPOL (Guayaquil, Ecuador) for helpful discussions.

\section{Literature Cited}

Bouhida, M., Lockhart, B. E., and Olszewski, N. E. 1993. An analysis of the complete sequence of a Sugarcane bacilliform virus genome infectious to banana and rice. J. Gen. Virol. 74:15-22.

Dean, F. B., Nelson, J. R., Giesler, T. L., and Lasken, R. S. 2001. Rapid amplification of plasmid and phage DNA using Phi29 DNA polymerase and multiply-primed rolling circle amplification. Genome Res. 11:1095-1099.

Dinkel, H., Van Roey, K., Michael, S., Davey, N. E., Weatheritt, R. J., Born, D., and Gibson, T. J. 2013. The eukaryotic linear motif resource ELM: 10 years and counting. Nucleic Acids Res. 42:D259-D266.

Geering, A. D. W., and Hull, R. 2012. Family caulimoviridae. Virus taxonomy: Ninth Report of the International Committee on Taxonomy of Viruses. Elsevier, London.

Gish, W., and States, D. J. 1993. Identification of protein coding regions by database similarity search. Nat. Genet. 3:266-272.

Higo, K., Ugawa, Y., Iwamoto, M., and Korenaga, T. 1999. Plant cis-acting regulatory DNA elements (PLACE) database: 1999. Nucleic Acids Res. 27: 297-300.

Kasteel, D. T. J., Perbal, M. C., Boyer, J. C., Wellink, J., Goldbach, R. W., Maule, A. J., and Van Lent, J. W. M. 1996. The movement proteins of Cowpea mosaic virus and Cauliflower mosaic virus induce tubular structures in plant and insect cells. J. Gen. Virol. 77:2857-2864

Kearse, M., Moir, R., Wilson, A., Stones-Havas, S., Cheung, M., Sturrock, S., and Drummond, A. 2012. Geneious Basic: An integrated and extendable desktop software platform for the organization and analysis of sequence data. Bioinformatics 28:1647-1649.

la Cour, T., Kiemer, L., Mølgaard, A., Gupta, R., Skriver, K., and Brunak, S. 2004 Analysis and prediction of leucine-rich nuclear export signals. Protein Eng. Des. Sel. 17:527-536.

Lam, E., and Chua, N. H. 1989. ASF-2: A factor that binds to the Cauliflower mosaic virus $35 \mathrm{~S}$ promoter and a conserved GATA motif in Cab promoters Plant Cell Online 1:1147-1156.

Laney, A. G., Hassan, M., and Tzanetakis, I. E. 2012. An integrated badnavirus is prevalent in fig germplasm. Phytopathology 102:1182-1189.

Larkin, M. A., Blackshields, G., Brown, N. P., Chenna, R., McGettigan, P. A., McWilliam, H., and Higgins, D. G. 2007. Clustal W and Clustal X version 2.0. Bioinformatics 23:2947-2948.

Lizardi, P. M., Huang, X., Zhu, Z., Bray-Ward, P., Thomas, D. C., and Ward, D. C 1998. Mutation detection and single-molecule counting using isothermal rolling-circle amplification. Nat. Genet. 19:225-232.

Lockhart, B. E. L. 1990. Evidence for a double-stranded circular DNA genome in a second group of plant viruses. Phytopathology 80:127-131.

Marchler-Bauer, A., and Bryant, S. H. 2004. CD-Search: Protein domain annotations on the fly. Nucleic Acids Res. 32:W327-W331.

Martin, R. R., Tzanetakis, I. E., Sweeney, M., and Wegener, L. 2006. A virus associated with blueberry fruit drop disease. Acta Hortic. 715:497-502.

Martin, R. R., Zhou, J., and Tzanetakis, I. E. 2011. Blueberry latent virus: An amalgam of the Partitiviridae and Totiviridae. Virus Res. 155:175-180.

Medberry, S. L., Lockhart, B. E., and Olszewski, N. E. 1992. The Commelina yellow mottle virus promoter is a strong promoter in vascular and reproductive tissues. Plant Cell Online 4:185-192.

Medberry, S. L., Lockhart, B. E. L., and Olszewski, N. E. 1990. Properties of Commelina yellow mottle virus's complete DNA sequence, genomic discontinuities and transcript suggest that it is a pararetrovirus. Nucleic Acids Res. 18:5505-5513.

Richert-Pöggeler, K. R., and Shepherd, R. J. 1997. Petunia vein-clearing virus: A plant pararetrovirus with the core sequences for an integrase function. Virology 236:137-146.

Song, J., Tan, H., Perry, A. J., Akutsu, T., Webb, G. I., Whisstock, J. C., and Pike, R. N. 2012. PROSPER: An integrated feature-based tool for predicting protease substrate cleavage sites. PLoS One 7:e50300.

Stamatakis, A. 2014. RAxML version 8: A tool for phylogenetic analysis and postanalysis of large phylogenies. Bioinformatics 30:1312-1313.

Xiong, Y., and Eickbush, T. H. 1990. Origin and evolution of retroelements based upon their reverse transcriptase sequences. EMBO J. 9:3353-3362.

Zhou, J., Bean, R. L., Vogt, V. M., and Summers, M. 2007. Solution structure of the Rous sarcoma virus nucleocapsid protein: $\mu \Psi$ RNA packaging signal complex. J. Mol. Biol. 365:453-467. 